\title{
A Multi-gene Genetic Programming Fuzzy Inference System for Regression Problems
}

\author{
Adriano S. Koshiyama ${ }^{1}$ Marley M.B.R. Vellasco ${ }^{1}$ and Ricardo Tanscheit ${ }^{1}$ \\ ${ }^{1}$ Department of Electrical Engineering, Pontifical Catholic University of Rio de Janeiro, \\ Rio de Janeiro, RJ, Brazil, e-mail: [adriano,marley,ricardo]@ele.puc-rio.br.
}

\begin{abstract}
This work presents a novel Genetic Fuzzy System (GFS), called Genetic Programming Fuzzy Inference System for Regression problems (GPFISRegress). It makes use of Multi-Gene Genetic Programming to build the premises of fuzzy rules, including t-norms, negation and linguistic hedge operators. GPFIS-Regress also defines a consequent term that is more compatible with a given premise and makes use of aggregation operators to weigh fuzzy rules in accordance with their influence on the problem. The system has been applied to a set of benchmarks and has also been compared to other GFSs, showing competitive results in terms of accuracy and interpretability.
\end{abstract}

Keywords: Genetic Fuzzy System, Genetic Programming, Regression.

\section{Introduction}

Regression problems are widely reported in the literature [1-3]. Generalized Linear Models, Neural Networks and Genetic Programming tend to provide solutions with high accuracy. However, high precision is not always associated to a reasonable linguistic comprehension, that is, it may be difficult to identify, in linguistic terms, the relation between the output and input variables. Fuzzy Inference Systems (FIS) are especially useful when reasonable accuracy and interpretability are desired [4].

As in regression problems a dataset is commonly available, supervised learning can be used to find the FIS parameters (rule base, granularity and membership functions shape, etc.). Genetic Fuzzy Systems (GFSs) $[5,6]$ are capable of doing that in an efficient way. As a GFS integrates a Fuzzy Inference System (FIS) and a Genetic Based Meta-Heuristic $(\mathrm{GBMH})$, it provides fair accuracy and linguistic interpretability (FIS component) through the automatic learning of its parameters/rules (GBMH component). Works on GFSs applied to regression problems are mostly based on improving the Genetic Based Meta-Heuristic counterpart of GFSs by using Multi-Objective Evolutionary Algorithms [3,7-10]. In general most of these works do not explore linguistic hedges and negation operators. Procedures for the selection of consequent terms have not been reported and few works weigh fuzzy rules. In ad- dition GFSs based on Genetic Programming have never been applied to regression problems.

This work presents a novel GFS called Genetic Programming Fuzzy Inference System for Regression problems (GPFIS-Regress). The main characteristics of this model are: $(i)$ it makes use of Multi-Gene Genetic Programming [2, 11], a Genetic Programming generalization that works on a single-objective framework, which in some situations can be more reliable computationally than multi-objective approaches; $(i i)$ it employs aggregation, negation and linguistic hedge operators in a simplified manner; ( iii) it applies some heuristics to define the consequent term best suited to a given antecedent term.

This paper is organized as follows: section 2 presents the main concepts of Multi-Gene Genetic Programming; section 3 presents the GPFISRegress model; case studies are dealt with in section 4 an section 5 concludes the work.

\section{Multi-Gene Genetic Programming}

Genetic Programming (GP) $[1,12]$ employs a population of individuals, each of them denoted by a tree structure that codifies a mathematical equation, which describes the relationship between the output $Y$ and a set of input variables $X_{j}(j=1, \ldots, J)$. Based on these ideas, Multi-Gene Genetic Programming (MGGP) [2,11] generalizes GP as it denotes an individual as a structure of trees, also called genes, that similarly receives $X_{j}$ and tries to predict $Y$ (Figure 1).

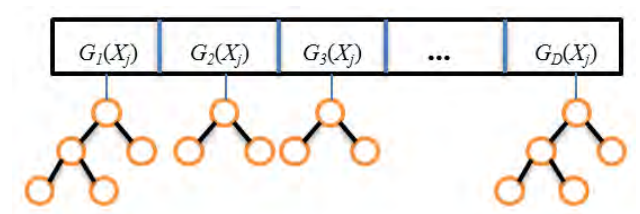

Figure 1: Example of multi-gene individual.

Each individual is composed of $D$ trees or functions $(d=1, \ldots, D)$ that relate $X_{j}$ to $Y$ through user-defined mathematical operations. It is easy to verify that MGGP generates solutions similar to those of GP when $D=1$. In GP terminology, the $X_{j}$ input variables are included in the Terminal Set, while the mathematical operations (plus, 
minus, etc.) are part of the Function Set (or Mathematical Operations Set).

With respect to genetic operators, mutation in MGGP is similar to that in GP. As for crossover, the level at which the operation is performed must be specified: it is possible to apply crossover at high and low levels. Figure 2a presents a multi-gene individual with five equations $(D=5)$ accomplishing a mutation, while Figure $2 \mathrm{~b}$ shows the low level crossover operation.

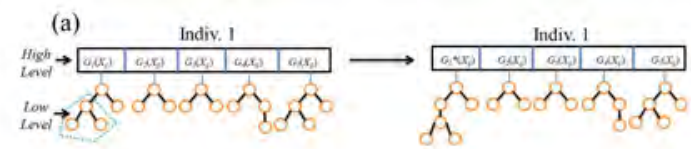

(b)
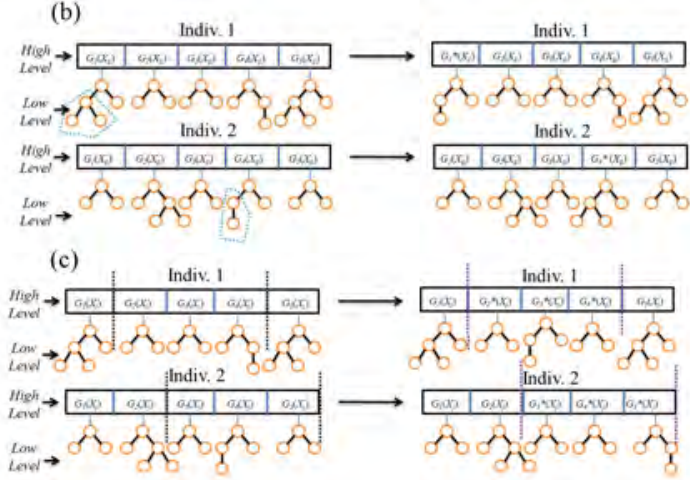

Figure 2: Application example of MGGP operators: (a) mutation; (b) low level crossover; and (c) high level crossover.

The low level is the space where it is possible to manipulate structures (Terminals and Mathematical Operations) of equations present in an individual. In this case, both operations are similar to those performed in GP. The high level, on the other hand, is the space where expressions can be manipulated in a macro way. An example of high level crossover is shown in Figure 2c. By observing the dashed lines it can be seen that the equations were switched from an individual to the other. The cutting point can be symmetric - the same number of equations is exchanged between individuals -, or asymmetric. Intuitively, high level crossover has a deeper effect on the output than low level crossover and mutation have.

In general, the evolutionary process in MGGP differs from that in GP due to the addition of two parameters: maximum number of trees per individual and high level crossover rate. A high value is normally used for the first parameter to assure a smooth evolutionary process. On the other hand, the high level crossover rate, similarly to other genetic operators rates, needs to be adjusted.

\section{GPFIS-Regress model}

GPFIS-Regress is a typical Pittsburgh-type GFS [5]. Its development begins with the mapping of crisp values into membership degrees to fuzzy sets (Fuzzification). Then, the fuzzy inference process is divided into three subsections: $(i)$ generation of fuzzy rule premises (Formulation); (ii) assignment of a consequent term to each premise (Premises Splitting) and (iii) aggregation of each activated fuzzy rule (Aggregation). Finally, Defuzzification and Evaluation are performed.

\subsection{Fuzzification}

In regression problems, the main information for predicting the behavior of an output $y_{i} \in Y(i=$ $1, \ldots, n)$ consists of its $J$ attributes or features $x_{i j} \in$ $X_{j}(j=1, \ldots, J)$. A total of $L$ fuzzy sets are associated to each $\mathrm{j}$-th feature and are given by $A_{l j}=$ $\left\{\left(x_{i j}, \mu_{A_{l j}}\left(x_{i j}\right)\right) \mid x_{i j} \in X_{j}\right\}$, where $\mu_{A_{l j}}: X_{j} \rightarrow[0,1]$ is a membership function that assigns to each observation $x_{i j}$ a membership degree $\mu_{A_{l j}}\left(x_{i j}\right)$ to a fuzzy set $A_{l j}$. Similarly, for $Y$ (output variable), $K$ fuzzy sets $B_{k}(k=1, \ldots, K)$ are associated.

Three aspects are taken into account when defining membership functions: $(i)$ form (triangular, trapezoidal, etc.); (ii) support set of $\mu_{A_{l j}}\left(x_{i j}\right)$; (iii) an appropriate linguistic term, qualifying the subspace constituted by $\mu_{A_{l j}}\left(x_{i j}\right)$ with a context-driven adjective. Ideally, these tasks should be carried out by an expert, whose knowledge would improve comprehensibility. In practice, it is not always easy to find a suitable expert. Therefore it is very common $[5,13]$ to define membership functions as shown in Figure 3.

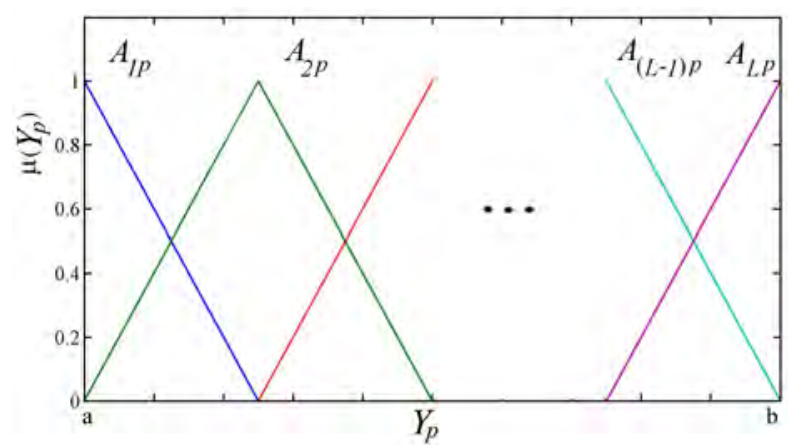

Figure 3: Membership functions for $x_{i j} \in X_{j}$ variables. For $Y$ read $A_{l j}$ as $B_{k}$.

\subsection{Fuzzy Inference}

\subsubsection{Formulation}

A fuzzy rule premise is commonly defined by:

"If $X_{1}$ is $A_{l 1}$ and $\ldots$ and $X_{j}$ is $A_{l j}$ and $\ldots$ and $X_{J}$
is $X_{l J}$ "

or, in mathematical terms:

$$
\mu_{A_{d}}\left(\mathbf{x}_{i}\right)=\mu_{A_{l 1}}\left(x_{i 1}\right) * \ldots * \mu_{A_{l J}}\left(x_{i J}\right)
$$

where $\mu_{A_{d}}\left(\mathbf{x}_{i}\right)=\mu_{A_{d}}\left(x_{i 1}, \ldots, x_{i J}\right)$ is the joint membership degree of the $\mathrm{i}$-th pattern $\mathbf{x}_{i}=\left[x_{i 1}, \ldots, x_{i J}\right]$ 
with respect to the d-th premise $(d=1, \ldots, D)$, computed by using a t-norm $*$. A premise can be elaborated by using t-norms, t-conorms, linguistic hedges and negation operators to combine the $\mu_{A_{l j}}\left(x_{i j}\right)$. As a consequence, the number of possible combinations grows as the number of variables, operators and fuzzy sets increase. Therefore, GPFIS-Regress employs MGGP to search for the most promising combinations, i.e., fuzzy rule premises. Figure 4 exemplifies a typical solution provided by MGGP.

For example, premise 1 represents: $\mu_{A_{1}}\left(\mathbf{x}_{i}\right)=$ $\mu_{A_{21}}\left(x_{i 1}\right) * \mu_{A_{32}}\left(x_{i 2}\right)$ and, in linguistic terms, "If $X_{1}$ is $A_{21}$ and $X_{2}$ is $A_{32}$ ". Let $\mu_{A_{d}}\left(\mathbf{x}_{i}\right)$ be the dth premise codified in the d-th tree of an MGGP individual. Table 1 presents the components used for reaching the solutions shown in Figure 4.

Table 1: Input Fuzzy Sets and Operators to generate solution in Figure 4.

\begin{tabular}{|c|c|}
\hline $\begin{array}{l}\text { Input Fuzzy Sets } \\
\text { (Terminals Set) }\end{array}$ & $\begin{array}{c}\text { Fuzzy Operators Set } \\
\text { (Functions Set) }\end{array}$ \\
\hline $\begin{array}{c}\mu_{A_{11}}\left(x_{i 1}\right), \ldots, \mu_{A_{L 1}}\left(x_{i 1}\right) \\
\ldots, \mu_{A_{l j}}\left(x_{i j}\right), \ldots, \\
\mu_{A_{L J}}\left(x_{i J}\right)\end{array}$ & $\begin{array}{c}\text { t-norm }(*) \text {, linguistic } \\
\text { hedge (dilatation operator } \\
-\sqrt{ }) \text { and classical negation } \\
\text { operator }\end{array}$ \\
\hline
\end{tabular}

In GPFIS-Regress, the set of $\mu_{A_{l j}}\left(x_{i j}\right)$ represents the Input Fuzzy Sets or, in GP terminology, the Terminal Set, while the Functions Set is replaced by the Fuzzy Operators Set. Thus MGGP is used for obtaining a set of fuzzy rules premises $\mu_{A_{d}}\left(\mathbf{x}_{i}\right)$. In order to fully develop a fuzzy rule base, it is necessary to find the consequent term (expressed in a MGGP individual) best suited to each $\mu_{A_{d}}\left(\mathbf{x}_{i}\right)$. This is performed in the premises splitting stage. It should be mentioned that the steps presented below - premises splitting, aggregation and evaluation - are repeated for every individual of the MGGP population.

\subsubsection{Premises Splitting}

There are two ways to define which consequent term is best suited to a fuzzy rule premise: $(i)$ allow a GBMH to perform this search (a common procedure in several works); or (ii) employ methods that directly draw information from the dataset so as to connect a premise to a consequent term. In GPFISRegress the second option has been adopted in order to prevent a premise with a large coverage in the dataset, or able to predict a certain region of the output, to be associated to an unsuitable consequent term. Instead of searching for all elements of a fuzzy rule, as a GBMH does, GPFIS-Regress measures the compatibility between $\mu_{A_{d}}\left(\mathbf{x}_{i}\right)$ and the consequent terms. This also promotes reduction of the search space.

In this sense, the Similarity Degree $\left(S D_{k}\right)$ between the $\mu_{A_{d}}\left(\mathbf{x}_{i}\right)$ and the consequent terms is em- ployed:

$$
S D_{k}=\min \left(1-\frac{\sum_{i=1}^{n}\left|\mu_{A_{d}}\left(\mathbf{x}_{i}\right)-\mu_{B_{k}}\left(y_{i}\right)\right|}{n}, I_{\{0,1\}}\right)
$$

where $\sum_{i=1}^{n}\left|\mu_{A_{d}}\left(\mathbf{x}_{i}\right)-\mu_{B_{k}}\left(y_{i}\right)\right|$ is the manhattan distance between the d-th premise and the $\mathrm{k}$-th consequent term, while $I_{\{0,1\}}$ is an indicator variable, which takes value 0 when $\mu_{A_{d}}\left(\mathbf{x}_{i}\right)=0, \forall i$, and 1 otherwise. When $\mu_{A_{d}}\left(\mathbf{x}_{i}\right)=\mu_{B_{k}}\left(y_{i}\right)$ for all $t$, then $F C D_{k}=1$, i.e., premise and consequent term are totally similar. A consequent term for $\mu_{A_{d}}\left(\mathbf{x}_{i}\right)$ is selected as the k-th consequent which maximize $S D_{k}$. A premise with $S D_{k}=0$, for all $k$, is not associated to any consequent term (and not considered as a fuzzy rule).

\subsubsection{Aggregation}

A premise associated to the $\mathrm{k}$-th consequent term (i.e., a fuzzy rule) is denoted by $\mu_{A_{d^{(k)}}}\left(\mathbf{x}_{i}\right)$, which, in linguistic terms, means: "If $X_{1}$ is $A_{l 1}$, and ..., and $X_{J}$ is $A_{l J}$, then $Y$ is $B_{k}$ ". Therefore, the whole fuzzy rule base is given by $\mu_{A_{1^{(k)}}}\left(\mathbf{x}_{i}\right), \ldots, \mu_{A_{D^{(k)}}}\left(\mathbf{x}_{i}\right)$, $\forall k=1, \ldots, K$. A new pattern $\mathbf{x}_{i}^{*}$ may have a non zero membership degree to several premises, associated either to the same or to different consequent terms. In order to generate a consensual value, the aggregation step tries to combine the activation degrees of all fuzzy rules associated to the same consequent term.

Consider $D^{(k)}$ as the number of fuzzy rules associated to $\mathrm{k}$-th consequent term $\left(d^{(k)}=\right.$ $\left.1^{(k)}, 2^{(k)} \ldots, D^{(k)}\right)$. Given an aggregation operator $g:[0,1]^{D^{(k)}} \rightarrow[0,1]$ (see [14]), the predicted membership degree of $\mathbf{x}_{i}^{*}$ to each $\mathrm{k}$-th consequent term $-\hat{\mu}_{B_{k}}\left(y_{i}^{*}\right)$ - is computed by:

$$
\begin{array}{r}
\hat{\mu}_{B_{1}}\left(y_{i}^{*}\right)=g\left[\mu_{A_{1(1)}}\left(\mathbf{x}_{i}^{*}\right), \ldots, \mu_{A_{D^{(1)}}}\left(\mathbf{x}_{i}^{*}\right)\right] \\
\hat{\mu}_{B_{2}}\left(y_{i}^{*}\right)=g\left[\mu_{A_{1(2)}}\left(\mathbf{x}_{i}^{*}\right), \ldots, \mu_{A_{D^{(2)}}}\left(\mathbf{x}_{i}^{*}\right)\right] \\
\ldots \\
\hat{\mu}_{B_{K}}\left(y_{i}^{*}\right)=g\left[\mu_{\left.A_{1(K)}\left(\mathbf{x}_{i}^{*}\right), \ldots, \mu_{A_{D^{(K)}}}\left(\mathbf{x}_{i}^{*}\right)\right]}\right.
\end{array}
$$

There are many aggregation operators available (e.g., see [14]), the Maximum being the most widely used [4]. Nevertheless other operators such as arithmetic and weighted averages may also be used. As for weighted arithmetic mean, it is necessary to solve a Restricted Least Squares problem (RLS) in order to establish the weights:

$$
\begin{array}{r}
\min : \sum_{i=1}^{n}\left(\hat{\mu}_{B_{k}}\left(y_{i}\right)-\sum_{d^{(k)}=1}^{D^{(k)}} w_{d^{(k)}} \mu_{A_{d^{(k)}}}\left(\mathbf{x}_{i}\right)\right)^{2} \\
\text { s.t. }: \sum_{d^{(k)}=1}^{D^{(k)}} w_{d^{(k)}}=1 \text { and } w_{d^{(k)}} \geq 0
\end{array}
$$

where $w_{d^{(k)}}$ is the weight or the influence degree of $\mu_{A_{d^{(k)}}}\left(\mathbf{x}_{i}\right)$ in the prediction of elements related 


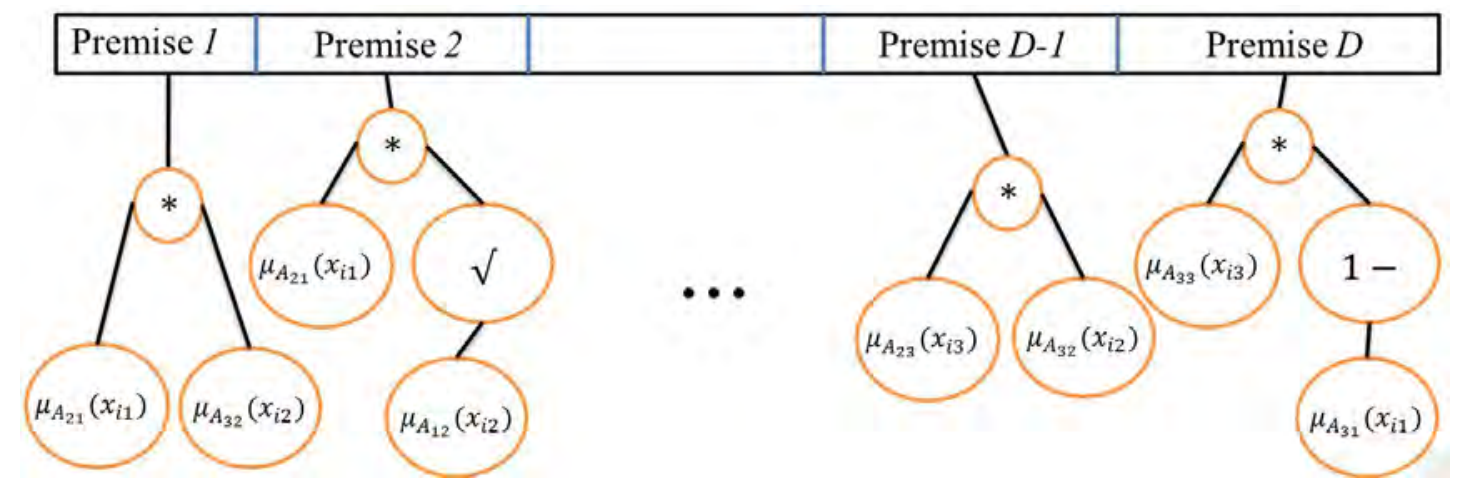

Figure 4: Example of fuzzy rule premises codified in an MGGP individual.

to the $\mathrm{k}$-th consequent term. This is a typical Quadratic Programming problem, the solution of which is easily computed by using algorithms discussed in [15]. This aggregation procedure is called by Weighted Average by Restricted Least Squares (WARLS).

\subsection{Defuzzification}

Proposition 1 Consider $y_{i} \in Y$, with $a \leq y_{i} \leq b$ where $a, b \in \mathbb{R}$, and, associated to $Y, K$ triangular membership functions, normal, 2-overlapped ${ }^{1}$ and strongly partitioned (identical to Figure 3). Then $y_{i}$ can be rewritten as:

$$
y_{i}=c_{1} \mu_{B_{1}}\left(y_{i}\right)+c_{2} \mu_{B_{2}}\left(y_{i}\right)+\ldots+c_{J} \mu_{B_{K}}\left(y_{i}\right)
$$

where $c_{1}, \ldots, c_{K}$ is the "center" $-\mu_{B_{k}}\left(c_{k}\right)=1-$ of each $k$-th membership function.

The proof can be found in [16]. This linear combination, which is a defuzzification procedure, is usually known as the Height Method. From this proposition, the following conclusions can be drawn:

1. If $\mu_{B_{k}}\left(y_{i}\right)$ is known, then $y_{i}$ is also known.

2. If only a prediction $\hat{\mu}_{B_{k}}\left(y_{i}\right)$ of $\mu_{B_{k}}\left(y_{i}\right)$ is known, such that $\sup _{y_{t}}\left|\mu_{B_{k}}\left(y_{i}\right)-\hat{\mu}_{B_{k}}\left(y_{i}\right)\right| \leq \epsilon$, when $\epsilon \rightarrow 0$ the defuzzification output $\hat{y}_{i}$ that approximates $y_{i}$ is given by:

$$
\hat{y}_{i}=c_{1} \hat{\mu}_{B_{1}}\left(y_{i}\right)+c_{2} \hat{\mu}_{B_{2}}\left(y_{i}\right)+\ldots+c_{K} \hat{\mu}_{B_{K}}\left(y_{t}\right)
$$

When $\hat{\mu}_{B_{k}}\left(y_{i}\right) \approx \mu_{B_{k}}\left(y_{i}\right)$ is not verified, the Mean of Maximum or the Center of Gravity [17] defuzzification methods may provide a better performance. However, due to the widespread use of strongly partitioned fuzzy sets in the experiments with GPFISRegress, a normalized version of the Height Method (8) has been employed:

$$
\hat{y}_{i}=\frac{c_{1} \hat{\mu}_{B_{1}}\left(y_{i}\right)+\ldots+c_{K} \hat{\mu}_{B_{K}}\left(y_{i}\right)}{\hat{\mu}_{B_{1}}\left(y_{i}\right)+\ldots+\hat{\mu}_{B_{K}}\left(y_{i}\right)}
$$

\footnotetext{
${ }^{1}$ a fuzzy set is normal if it has some element with maximum membership equal to 1 . Also, fuzzy sets are 2overlapped if $\min \left(\mu_{B_{u}}\left(y_{i}\right), \mu_{B_{z}}\left(y_{i}\right), \mu_{B_{v}}\left(y_{i}\right)\right)=0, \forall u, v, z \in$ $k=1, \ldots, K$
}

It is now possible to evaluate an individual of GPFIS-Regress by using $\hat{y}_{i}$.

\subsection{Evaluation}

The Evaluation procedure in GPFIS-Regress is defined by a primary objective - error minimization and a secondary objective - complexity reduction. The primary objective is responsible for ranking individuals in the population, while the secondary one is used as a tiebreaker criteria.

A simple fitness function for regression problems is the Mean Squared Error $(M S E)$ :

$$
M S E=\frac{\sum_{i=1}^{n}\left(y_{i}-\hat{y}_{i}\right)^{2}}{2 * n}
$$

The best individual in the population is the solution which minimizes equation (10). GPFISRegress tries to reduce the complexity of the rule base by employing a simple heuristic: Lexicographic Parsimony Pressure [18]. This technique is only used in the selection phase: given two individuals with the same fitness, the best one is that with fewer nodes. Fewer nodes indicate rules with fewer antecedent elements, linguistic hedges and negation operators, as well as few premises $\left(\mu_{A_{d}}\left(\mathbf{x}_{i}\right)\right)$, and, therefore, a small fuzzy rule set. After evaluation, a set of individuals is selected (through a tournament procedure) and recombined. This process is repeated until a stopping criteria is met. When this occurs, the final population is returned.

\section{Case Studies}

\subsection{Experiments Description}

Among the SFGs designed for solving regression problems, the Fast and Scalable Multi-Objective Genetic Fuzzy System (FS-MOGFS) [3] has been used in the experiments. In contrast to other works [7-10,19], FS-MOGFS has been chosen because:

1. it makes use of 17 datasets; five of them are highly scalable and high-dimensional;

2. it presents a comparison between three different GFSs; 
3. it describes in detail the parameters used for each model and the number of evaluations. Furthermore, results show accuracy (training and test sets) and rule base compactness (average number of rules and of antecedents elements per rule).

In its basic version, FS-MOGFS consists of:

- Each chromosome $(C)$ has two parts $\left(C=C_{1} \cup\right.$ $C_{2}$ ): $C_{1}$ represents the number of triangular and uniformly distributed membership functions and $C_{2}=\left[\alpha_{1}, \alpha_{2}, \ldots, \alpha_{J}\right]$, where each $\alpha_{j}$ is a degree of displacement of the j-th variable [19]. To obtain the best possible values for $C$, the model incorporates a Multi-Objective Genetic Algorithm (MOGA) based on SPEA2 [3]. The two objectives are: minimize the Mean Squared Error and the number of rules.

- In order to build the complete knowledge base (rules and membership functions), rule extraction via Wang \& Mendel's algorithm is performed for each chromosome. [20]. The Mamdani-type SIF employs the minimum for t-norm and implication, and center of gravity for defuzzification.

Extensions of FS-MOGFS have resulted in two other models: (i) FS-MOGFS ${ }^{e}$ - identical to FSMOGFS, but with fast error computation by leaving aside a portion of the database; (ii) FSMOGFS+TUN: similar to the previous one, but with fine tuning of membership functions parameters [21]. This model provided the best results and was therefore used for comparison with GPFISRegress. Databases shown in Table 2 [3] have been considered in case studies.

Table 2: Databases considered in experiments.

\begin{tabular}{cccc}
\hline \hline Database & Acronym & $J$ & $n$ \\
\hline Electrical Maintenance & ELE & 4 & 1056 \\
Auto MPG6 & MPG6 & 5 & 398 \\
Auto MPG8 & MPG8 & 7 & 398 \\
Analcat & ANA & 7 & 4052 \\
Abalone & ABA & 8 & 4177 \\
Stock & STP & 9 & 950 \\
Weather Izmir & WIZ & 9 & 1461 \\
Weather Ankara & WAN & 9 & 1609 \\
Forest Fires & FOR & 12 & 51 \\
Mortgage & MOR & 15 & 1049 \\
Treasury & TRE & 15 & 1049 \\
Baseball & BAS & 16 & 337 \\
MV Artificial Domain & MV & 10 & 40768 \\
Elevators & ELV & 18 & 16559 \\
Computer-Activity & CA & 21 & 8192 \\
Ailerons & AIL & 40 & 13750 \\
The Insurance Company & TIC & 85 & 9822 \\
\hline
\end{tabular}

Five of the 17 databases are of high dimensionality: ELV, AIL, MV, CA e TIC; they have been obtained from the KEEL repository [3]. Similarly to the procedure adopted in Alcalá et al. [3], 100,000 evaluations (population size $=100$ and number of generations $=1000$ ) have been carried out in each execution. The remaining parameters are shown in Table 3. With 6 repeats of 5 -fold cross-validation, the GPFIS-Regress was executed 30 times. The metrics shown for each database are the average for the 30 trained models. The Mean Squared Error has been used as the fitness function [3].

Table 3: GPFIS-Regress main configuration.

\begin{tabular}{cc}
\hline \hline Parameter & Value \\
\hline Population Size & 100 \\
Number of Generations & 1000 \\
Tree Maximum Depth & 5 \\
Tournament Size & 2 \\
High Level Crossover Rate & $50 \%$ \\
Low Level Crossover Rate & $85 \%$ \\
Mutation Rate & $15 \%$ \\
Elitism Rate & $1 \%$ \\
\hline Input Fuzzy Sets & 5 fuzzy sets, displayed \\
& like Figure 3 \\
Fuzzy Operators & Product, Classical \\
& Negation and \\
Partitioning Method & Square-Root \\
Aggregation Operator & SD \\
Defuzzification & WARLS \\
\hline \hline
\end{tabular}

It should be noted that preliminary tests considered three, five and seven fuzzy sets. As the results did not show any relevant difference in terms of accuracy, five strongly partitioned fuzzy sets (Figure 3) have been used throughout the experiments, as stated in Table 3 . In addition to FS-MOGFS+TUN [3], three other SFGs have been used for comparison: GR-MF [22],GA-WM [23] and GLD-WM [19].

Statistical analysis have followed recommendations from [3] and have been performed in the KEEL software [24], with a significance level of 0.1 .

\subsection{Results and Discussion}

Table 4 shows the results for all GFSs for each database in terms of MSE, average number of rules and of antecedent elements per rule. Results for models other than GPFIS-Regress have been taken from [3]. In general GPFIS-Regress has provided better results in $58 \%$ of cases, followed by FSMOGFS+TUN with 23\%. GLD-WM has performed better for one single database; the remaining SFGs performed below those three. In highdimensional problems, GPFIS-Regress has attained better results for three of the five databases.

Table 5 presents results for the Friedman test and Holm method for low-dimensional databases, given a significance level of $10 \%$ [3]. As GPFIS-Regress presented the lowest rank (1.5417), it was chosen as the reference model. It can be observed that GPFIS-Regress achieved higher accuracy than GRMF, GA-WM and GLD-WM have ( $\mathrm{p}$-value < 0.05). This has not been verified for GPFIS-Regress and FS-MOGFS+TUN (p-value > 0.10).

As far as GPFIS-Regress and FS-MOGFS+TUN are concerned, the former has achieved better results for 10 of the 17 databases, with two ties. The signal test has shown that the differences in results were not significant $(S=10, \mathrm{p}$-value $=0.3018)$. This may be due to the ties and to the small number of databases considered. As for rule base complexity, it can be noted that GPFIS-Regress obtained the most compact one in $53 \%$ of cases. 
Table 4: Results for GPFIS-Regress and other GFSs in termos of EQM. $\sigma=$ standard deviation of EQM. Results for Tr./Ts. (Training/Test) must be multiplied by $10^{5}, 10^{-5}$ and $10^{-8}$ for BAS, ELV and AIL respectively. R/A - Averages of Rules and Antecedent Elements per Rule.

\begin{tabular}{|c|c|c|c|c|c|c|c|c|c|c|c|c|c|c|c|}
\hline \multirow{2}{*}{$\begin{array}{c}\text { Data } \\
\text { Set }\end{array}$} & \multicolumn{3}{|c|}{ GR-MF } & \multicolumn{3}{|c|}{ GA-WM } & \multicolumn{3}{|c|}{ GLD-WM } & \multicolumn{3}{|c|}{ FS-MOGFS+TUN } & \multicolumn{3}{|c|}{ GPFIS-Regress } \\
\hline & $\mathrm{R} / \mathrm{A}$ & Tr. & Ts. & $\mathrm{R} / \mathrm{A}$ & Tr. & Ts. & $\mathrm{R} / \mathrm{A}$ & Tr. & Ts. & $\mathrm{R} / \mathrm{A}$ & Tr. & Ts. & $\mathrm{R} / \mathrm{A}$ & Tr. & Ts. \\
\hline $\begin{array}{c}\text { ELE } \\
\sigma\end{array}$ & $97 / 4$ & $\begin{array}{c}16645 \\
2319\end{array}$ & $\begin{array}{c}18637 \\
3386\end{array}$ & $47 / 4$ & $\begin{array}{c}17230 \\
2501\end{array}$ & $\begin{array}{c}18977 \\
3195\end{array}$ & $33 / 4$ & $\begin{array}{c}11483 \\
1085\end{array}$ & $\begin{array}{c}13384 \\
1978\end{array}$ & $9 / 2$ & $\begin{array}{c}8803 \\
739\end{array}$ & $\begin{array}{l}\mathbf{9 8 4 2} \\
1391\end{array}$ & $16 / 3$ & $\begin{array}{c}14695 \\
815\end{array}$ & $\begin{array}{c}16818 \\
493\end{array}$ \\
\hline MPG6 & $243 / 5$ & 1.423 & 28.933 & $186 / 5$ & 1.879 & 8.824 & $82 / 5$ & 2.294 & 4.387 & $22 / 3$ & 2.778 & 4.548 & $18 / 3$ & 2.890 & 4.003 \\
\hline$\sigma$ & & 0.073 & 8.633 & & 0.235 & 6.079 & & 0.249 & 0.899 & & 0.220 & 1.047 & & 0.066 & 0.336 \\
\hline MPG8 & $262 / 7$ & 1.356 & 49.36 & $214 / 7$ & 1.563 & 15.216 & $135 / 7$ & 1.709 & 4.782 & $24 / 3$ & 2.725 & 4.381 & $18 / 3$ & 2.780 & 4.087 \\
\hline$\sigma$ & & 0.104 & 16.2 & & 0.183 & 9.13 & & 0.170 & 1.445 & & 0.294 & 0.909 & & 0.091 & 0.116 \\
\hline ANA & $148 / 7$ & 0.005 & 0.017 & $150 / 7$ & 0.003 & 0.008 & $92 / 7$ & 0.006 & 0.008 & $17 / 3$ & 0.003 & 0.003 & $8 / 3$ & 0.002 & 0.003 \\
\hline$\sigma$ & & 0.001 & 0.008 & & 0.001 & 0.005 & & 0.001 & 0.004 & & 0.000 & 0.001 & & 0.000 & 0.000 \\
\hline$\overline{\mathrm{ABA}}$ & $498 / 8$ & 2.358 & 2.885 & $143 / 8$ & 2.433 & 2.549 & $31 / 8$ & 2.487 & 2.545 & $10 / 3$ & 2.393 & 2.454 & $16 / 3$ & 2.370 & 2.425 \\
\hline$\sigma$ & & 0.052 & 0.263 & & 0.052 & 0.163 & & 0.078 & 0.170 & & 0.092 & 0.163 & & 0.068 & 0.154 \\
\hline STP & $343 / 9$ & 0.4 & 1.543 & $344 / 9$ & 0.389 & 2.192 & $217 / 9$ & 0.299 & 0.435 & $25 / 3$ & 0.724 & 0.892 & $17 / 3$ & 1.143 & 1.283 \\
\hline$\sigma$ & & 0.019 & 2.484 & & 0.017 & 3.168 & & 0.025 & 0.067 & & 0.112 & 0.154 & & 0.173 & 0.256 \\
\hline WIZ & $331 / 9$ & 1.176 & 9.602 & $218 / 9$ & 1.233 & 3.529 & $107 / 9$ & 0.926 & 1.150 & $15 / 3$ & 0.867 & 1.011 & $17 / 3$ & 0.811 & 0.878 \\
\hline$\sigma$ & & 0.077 & 8.879 & & 0.065 & 4.023 & & 0.041 & 0.123 & & 0.040 & 0.177 & & 0.046 & 0.040 \\
\hline WAN & $397 / 9$ & 1.406 & 7.381 & $279 / 9$ & 1.522 & 2.82 & $133 / 9$ & 1.111 & 2.075 & $11 / 2$ & 1.313 & 1.581 & $15 / 2$ & 1.307 & 1.367 \\
\hline$\sigma$ & & 0.067 & 5.404 & & 0.065 & 2.825 & & 0.077 & 1.407 & & 0.174 & 0.580 & & 0.079 & 0.092 \\
\hline FOR & $396 / 12$ & 113 & 3300 & $395 / 12$ & 47 & 3693 & $377 / 12$ & 49 & 3847 & $33 / 3$ & 1593 & 2406 & $8 / 3$ & 1476 & 2446 \\
\hline$\sigma$ & & 17 & 2207 & & 24 & 2787 & & 18 & 2714 & & 570 & 2161 & & 868 & 2456 \\
\hline MOR & $209 / 15$ & 0.03 & 0.176 & $160 / 15$ & 0.02 & 0.093 & $78 / 15$ & 0.016 & 0.022 & $9 / 3$ & 0.015 & 0.018 & $15 / 3$ & 0.013 & 0.015 \\
\hline$\sigma$ & & 0.002 & 0.28 & & 0.003 & 0.147 & & 0.002 & 0.005 & & 0.004 & 0.012 & & 0.001 & 0.002 \\
\hline TRE & $189 / 15$ & 0.066 & 0.144 & $136 / 15$ & 0.045 & 0.064 & $70 / 15$ & 0.033 & 0.045 & $11 / 3$ & 0.030 & 0.040 & $15 / 3$ & 0.031 & 0.037 \\
\hline$\sigma$ & & 0.011 & 0.191 & & 0.007 & 0.046 & & 0.005 & 0.015 & & 0.004 & 0.012 & & 0.002 & 0.002 \\
\hline BAS & $262 / 16$ & 0.255 & 12.439 & $262 / 16$ & 0.202 & 11.706 & $244 / 16$ & $\begin{array}{l}0.138 \\
0.014\end{array}$ & $\begin{array}{l}3.610 \\
0.621\end{array}$ & $21 / 6$ & $\begin{array}{l}1.305 \\
0.172\end{array}$ & $\begin{array}{l}\mathbf{2 . 6 9 9} \\
0.620\end{array}$ & $16 / 3$ & $\begin{array}{l}1.469 \\
0.067\end{array}$ & $\begin{array}{l}3.037 \\
0.246\end{array}$ \\
\hline & & 0.02 & 2.177 & & 0.031 & 2.562 & & 0.014 & 0.621 & & 0.172 & 0.620 & & 0.067 & 0.246 \\
\hline MV & - & - & - & - & - & - & - & - & - & $16 / 3$ & 0.159 & 0.160 & $10 / 3$ & 2.607 & 2.599 \\
\hline$\sigma$ & - & - & - & - & - & - & - & - & - & & 0.031 & 0.032 & & 1.499 & 1.490 \\
\hline ELV & - & - & - & - & - & - & - & - & - & $8 / 3$ & 0.900 & 0.900 & $12 / 3$ & 0.875 & 0.886 \\
\hline$\sigma$ & - & - & - & - & - & - & - & - & - & & 0.200 & 0.200 & & 0.106 & 0.108 \\
\hline $\mathrm{CA}$ & - & - & - & - & - & - & - & - & - & $15 / 5$ & 4.763 & 5.063 & $15 / 4$ & 4.885 & 5.060 \\
\hline$\sigma$ & - & - & - & - & - & - & - & - & - & & 0.404 & 0.760 & & 0.729 & 0.739 \\
\hline AIL & - & - & & - & - & - & - & - & - & $20 / 4$ & 1.864 & 1.905 & $17 / 3$ & 1.829 & 1.858 \\
\hline$\sigma$ & - & - & - & - & - & - & - & - & - & & 0.221 & 0.233 & & 0.003 & 0.003 \\
\hline TIC & & - & & - & - & - & - & - & - & $25 / 7$ & 0.026 & 0.027 & $11 / 4$ & 0.026 & 0.027 \\
\hline$\sigma$ & - & - & - & - & - & - & - & - & - & & 0.000 & 0.002 & & 0.000 & 0.001 \\
\hline
\end{tabular}

Regarding interpretability and implementation, GPFIS-Regress has an advantage over FS-MOGFS+TUN in aspects such as: $(i)$ makes no change to membership functions parameters; (ii) employs a genetic-based meta-heuristic with a single objective, while FS-MOGFS+TUN does a multi-objective search.

Table 5: Results for Friedman test and Holm method.

\begin{tabular}{c|ccc}
\hline \hline $\mathrm{i}$ & Model & Rank & \\
\hline 4 & GR-MF & 4.6667 & \\
3 & GA-WM & 4.1250 & \\
2 & GLD-WM & 2.8750 & \\
& FS- & & \\
& MOGFS+TUN & 1.7917 & \\
& & & \\
& GPFIS- & & \\
& Regress & $\mathbf{1 . 5 4 1 7}$ & \\
& & & \\
\hline \hline Test & p-value & & \\
\hline \hline Friedman & $<0.0001$ & & \\
\hline \multirow{2}{*}{ Method } & $z=$ & \multirow{2}{*}{ p-value } \\
& $\left(R_{0}-R_{i}\right) / S E$ & & \\
\hline GR-MF & 4.8412 & $<0.0001$ & 0.0250 \\
GA-WM & 4.0020 & $<0.0001$ & 0.0333 \\
GLD-WM & 2.0655 & 0.0388 & 0.0500 \\
FS-MOGFS+TUN & 0.3872 & 0.6985 & 0.1000 \\
\hline \hline
\end{tabular}

\section{Conclusion}

This work has presented a novel Genetic Fuzzy System for solving regression problems, called GPFISRegress, which makes use of Multi-Gene Genetic Programming and a novel way to formulate the Fuzzy Reasoning Method (Formulation-SplittingAggregation). GPFIS-Regress has been compared to four other Genetic Fuzzy Systems for 17 datasets of low and high dimensionality. Results have shown the potentialities of the proposed approach.

Further developments and experiments shall include: $(i)$ evaluation of other t-norm, negation and linguistic hedges operators, as well as the use of tconorms in rules premises; $(i i)$ new splitting methods (through other similarity measures) and application of the Restricted Least Squares procedure with some adaptation to associate a more suitable consequent term to a given premise; (iii) evaluation of other aggregation operators, such as nonlinear ones (weighted geometric mean, etc.); this may provide better results mostly in terms of accuracy.

\section{References}

[1] R. Poli, W. B. Langdon, and N. F. McPhee. A Field Guide to Genetic Programming. Lulu.com, Rayleigh, 2008.

[2] A. H. Gandomi and A. H. Alavi. A new multigene genetic programming approach to nonlinear system modeling. Neural Computing and Applications, 21(1):171-187, 2012.

[3] R. Alcalá, M. J. Gacto, and F. Herrera. A fast and scalable multiobjective genetic fuzzy system for linguistic fuzzy modeling in highdimensional regression problems. Fuzzy Systems, IEEE Transactions on, 19(4):666-681, 2011.

[4] W. Pedrycz and F. Gomide. An introduction to fuzzy sets: analysis and design. MIT Press, Massachussets, 1998.

[5] F. Herrera. Genetic fuzzy systems: taxonomy, current research trends and prospects. Evolutionary Intelligence, 1(1):27-46, 2008. 
[6] M. Fazzolari, R. Alcala, Y. Nojima, H. Ishibuchi, and F. Herrera. A review of the application of multiobjective evolutionary fuzzy systems: Current status and further directions. IEEE Transactions on Fuzzy Systems, 21(1):45-65, 2013.

[7] M. Antonelli, P. Ducange, B. Lazzerini, and F. Marcelloni. Learning concurrently partition granularities and rule bases of mamdani fuzzy systems in a multi-objective evolutionary framework. International Journal of Approximate Reasoning, 50(7):1066-1080, 2009.

[8] P. Pulkkinen and H. Koivisto. A dynamically constrained multiobjective genetic fuzzy system for regression problems. IEEE Transactions on Fuzzy Systems, 18(1):161-177, 2010.

[9] A. A. Márquez, F. A. Márquez, A. M. Roldán, and A. Peregrín. An efficient adaptive fuzzy inference system for complex and high dimensional regression problems in linguistic fuzzy modelling. Knowledge-Based Systems, $54(0): 42-52,2013$.

[10] A. D. Benítez and J. Casillas. Multi-objective genetic learning of serial hierarchical fuzzy systems for large-scale problems. Soft Computing, 17(1):165-194, 2013.

[11] D. Searson, M. Willis, and G. Montague. Coevolution of nonlinear pls model components. Journal of Chemometrics, 21(12):592603, 2007.

[12] J. R. Koza. Genetic Programming: On the Programming of Computers by Means of Natural Selection. MIT Press, Massachusetts, 1992.

[13] H. Ishibuchi, M. Yamane, and Y. Nojima. Rule weight update in parallel distributed fuzzy genetics-based machine learning with data rotation. In In IEEE International Conference on Fuzzy Systems, pages 1-8. IEEE, 2013.

[14] G. Beliakov, A. Pradera, and T. Calvo. Aggregation functions: A guide for practitioners. Springer, Heidelberg, 2008.

[15] B. Schölkopf and A. J. Smola. Learning with Kernels: Support Vector Machines, Regularization, Optimization, and Beyond. MIT Press, Massachussets, 2001.
[16] W. Pedrycz. Granular Computing: Analysis and Design of Intelligent Systems. CRC Press, Boca Raton, 2013.

[17] Shounak Roychowdhury and Witold Pedrycz. A survey of defuzzification strategies. International Journal of Intelligent Systems, 16(6):679-695, 2001.

[18] Sean Luke and Liviu Panait. Lexicographic parsimony pressure. In GECCO 2002: Proceedings of the Genetic and Evolutionary Computation Conference, pages 829-836, New York, 2002.

[19] Rafael Alcalá, Jesús Alcalá-Fdez, Francisco Herrera, and José Otero. Genetic learning of accurate and compact fuzzy rule based systems based on the 2-tuples linguistic representation. International Journal of Approximate Reasoning, 44(1):45-64, 2007.

[20] L. X. Wang and J. M. Mendel. Generating fuzzy rules by learning from examples. IEEE Transactions on Systems, Man and Cybernetics, 22(6):1414-1427, 1992.

[21] M. J. Gacto, R. Alcalá, and F. Herrera. Adaptation and application of multi-objective evolutionary algorithms for rule reduction and parameter tuning of fuzzy rule-based systems. Soft Computing, 13(5):419-436, 2008.

[22] O. Cordón, F. Herrera, and P. Villar. Generating the knowledge base of a fuzzy rulebased system by the genetic learning of the data base. IEEE Transactions on Fuzzy Systems, 9(4):667-674, 2001.

[23] O. Cordón, F. Herrera, L. Magdalena, and P. Villar. A genetic learning process for the scaling factors, granularity and contexts of the fuzzy rule-based system data base. Information Sciences, 136(1-4):85-107, 2001.

[24] J. Alcalá-Fdez, A. Fernandez, J. Luengo, J. Derrac, S. García, L. Sánchez, and F. Herrera. Keel data-mining software tool: Data set repository, integration of algorithms and experimental analysis framework. Journal of Multiple-Valued Logic and Soft Computing, 17(2-3):255-287, 2011. 